\title{
Modelling route for abradable coatings
}

\author{
H.I. Faraoun ${ }^{\text {a }}$, J.L. Seichepine ${ }^{\text {a }}$, C. $\operatorname{Coddet}^{\text {a }}$, H. Aourag ${ }^{\text {a }}$, J. Zwick ${ }^{\text {b }}$, N. Hopkins ${ }^{c}$, \\ D. Sporer ${ }^{\mathrm{d}}$, M. Hertter ${ }^{\mathrm{e}}$ \\ a Laboratoire d'Etudes et de Recherches sur les Matériaux, les Procédés et les Surfaces. LERMPS-UTBM, France \\ b Surface Engineering Institute, Aachen University, Germany \\ ${ }^{\mathrm{c}}$ Rolls-Royce plc, Derby, United Kingdom \\ d Sulzer-Metco (Canada) Inc, Fort Saskatchewan, Canada \\ e MTU Aero Engines, München, Germany
}

\begin{abstract}
Improving sealing between rotating and stationary parts in aerospace gas turbines significantly increases engine performance by improving thermal efficiencies. To reach this aim, abradable seals are being incorporated into turbine casings. With an abradable seal, the blade tips incur into the shroud, thereby reducing the gap between the rotor and the coating to a minimum. These coatings are generally multiphase materials applied by thermal spray techniques and consisting in a combination of metallic matrix and additional dislocator phases with a controlled amount of porosity. The sealing effectiveness requires a combination of properties that are usually optimised empirically with thermal spray coatings generally made up from a range of two-phase powder mixtures.

The present study intends to initiate a theoretical approach for the study of these materials aiming at developing a prediction strategy for structure improvement. Image analyses and finite element calculations were used to examine the effect of phase morphology on the mechanical behaviour of two reference abradable systems, namely AlSi-hBN and NiCrAl-Bentonite for compressor stages. Scanning Electronic Microscopy (SEM) was used to obtain a series of micrographs for coating characterisation. These micrographs were then treated to create equivalent images based on geometrical description of the inherent morphology. The resultant reduced images are used to carry out finite element calculations, in order to determine the mechanical properties of each coating. It is believed that this approach provides consistent results and is believed to be a reliable starting point for further coatings design.
\end{abstract}

Keywords: Wear protection; Finite element analysis; Thermal spray; Abradable coatings

\section{Introduction}

Progress in the requirements of air transportation is imposing increasing demands to improve both operating and new industrial turbines for jet engines, i.e. increasing efficiency and power output. One of the critical areas being addressed is to close the gap between the blade tip and shroud thereby reducing the parasitic leakage flows through the various seals [1-3]. Abradable seals are being developed in various turbine locations [4]. In an abradable seal system, the blade tip incurs into the shroud, thereby reducing the tip gap to a minimum. There exist several types of abradable materials that can be manufactured by a number of methods [5]. However, thermal spraying is the most popular choice for processing abradable seal materials [4]. Along with its wide-range applicability, thermal spraying makes the operation of restoration of the seals efficient, which is a critical need in aero-engine industry. During service, the abradable material is worn-in by the blade-tip with quite complex wear mechanisms involved. This includes cutting, smearing, adhesive transfer, crushing, melting, erosion and oxidation [6]. Preferably, the abradable must not damage the incurring tip and remain reasonably smooth after the rub. Good abradability implies then a reasonable softness, but also a sufficient hardness to resist against the erosion due to the high-speed gas flow and the particles it contains. Oxidation and corrosion resistance, resistance to thermal shock and spontaneous combustion as well as debris compatibility are also key criteria that the seal 
has to fulfil for it is an engine system. To answer to all these requirements, the abradable materials often consist of a composite mixture of two or three structural components: metallic matrix and a second phase as solid lubricant with a controlled amount of porosity [7]. The solid lubricant is a second phase designed to shear easily. Entrapped within the metallic matrix, it helps to minimise the coating's material transfer to the blade and also to reduce the size of the debris.

The development of abradable materials composition and structure has been mostly empirical by means of test rigs [8$10]$ and tribometers $[8,11]$ supplying an overview of the tribosystem under investigation and allowing to plot wear maps, a useful tool that helps to determine under which conditions specific wear mechanisms are dominant $[12,13]$. With this information, the design can be steered by comparison of the in-service behaviours of various abradable materials.

The materials engineer requires a model to assist him in his task. Nevertheless, the theoretical models for abradable materials resume to some schematics attempting to explain the wear mechanism established on the basis of empirical observation. Schmid et al. [9] suggest a schematic intended to be used for the design of the high temperature abradable model. To our knowledge, no more elaborated theoretical studies of the structure of abradable coatings exist; though it is believed that their comprehension requires a good description of the volumes, distributions and sizes of the porosities and second phase particles.

In this paper, an attempt is made to present a model that can serve as a recipe for a theoretical investigation of structure improvement for abradable seals design. This work is a part of a wider research activity, carried out in the framework of a European Contract [14] whose goal is to develop abradable materials with increased service life. Much attention is assigned to the microstructure and its impact on the mechanical behaviour of coating. The first task was to gain further understanding on the abradability-structure relationship, which was the subject of a former paper [15]. An image analysis procedure was developed to quantify the different geometrical parameters of the structural elements of the abradable coatings. This description is used here to quantitatively estimate the mechanical properties of two abradable coatings obtained by thermal spraying.

The present paper is organised as follows. Section 2 presents the selection of the two abradable coatings and experimental details used for the microstructure characterisation. Section 3 concentrates on the specific quantitative image analysis procedure developed for abradable coatings by means of equivalent images. Section 4 introduces a finite element model aimed at initiating a comprehensive study for the correlation between the microstructure of the coating and its mechanical law of behaviour. The paper is then concluded by a summary of the most important points to be taken into account for further enhancement of the model presented.

\section{Material selection and experimental procedure for characterisation}

Abradable seals are usually classified according to temperature capabilities. In this paper, attention will be focused on two abradable coatings obtained by thermal spraying techniques. The first one, AlSi-hBN (powder supplied by Sulzer Metco under the reference SM 320), is a metal matrix-solid lubricant coating aimed at operating for temperatures up to $480{ }^{\circ} \mathrm{C}$ [16]. It was produced by means of the Air Plasma Spray (APS) deposition technique using a Plasma-Technik PT F4 gun. The second one, NiCrAl-Bentonite (powder supplied by Sulzer Metco under the reference SM 314), is a metal matrix-dislocator coating designed for a maximum temperature of $850{ }^{\circ} \mathrm{C}$. It was deposited by the combustion spray process $[18,19]$ using a Metco 6PII gun. Observations were carried out by Scanning Electron Microscopy (SEM) using either a Phillips XL-30 or a ZEISS DSM 982 Gemini apparatus. Conventional mechanical polishing metallographic techniques were used after the samples were mounted in a low viscosity resin. The coating images were essentially taken under backscattering electron imaging conditions to reveal, thanks to their different chemical compositions, their different structural elements.

Fig. 1 compares typical micrographs obtained for the two coatings. They show that the scales of the "structural elements" constituting the two coatings are fairly different. Table 1 gives the volume fraction of metallic matrix, second phase particles
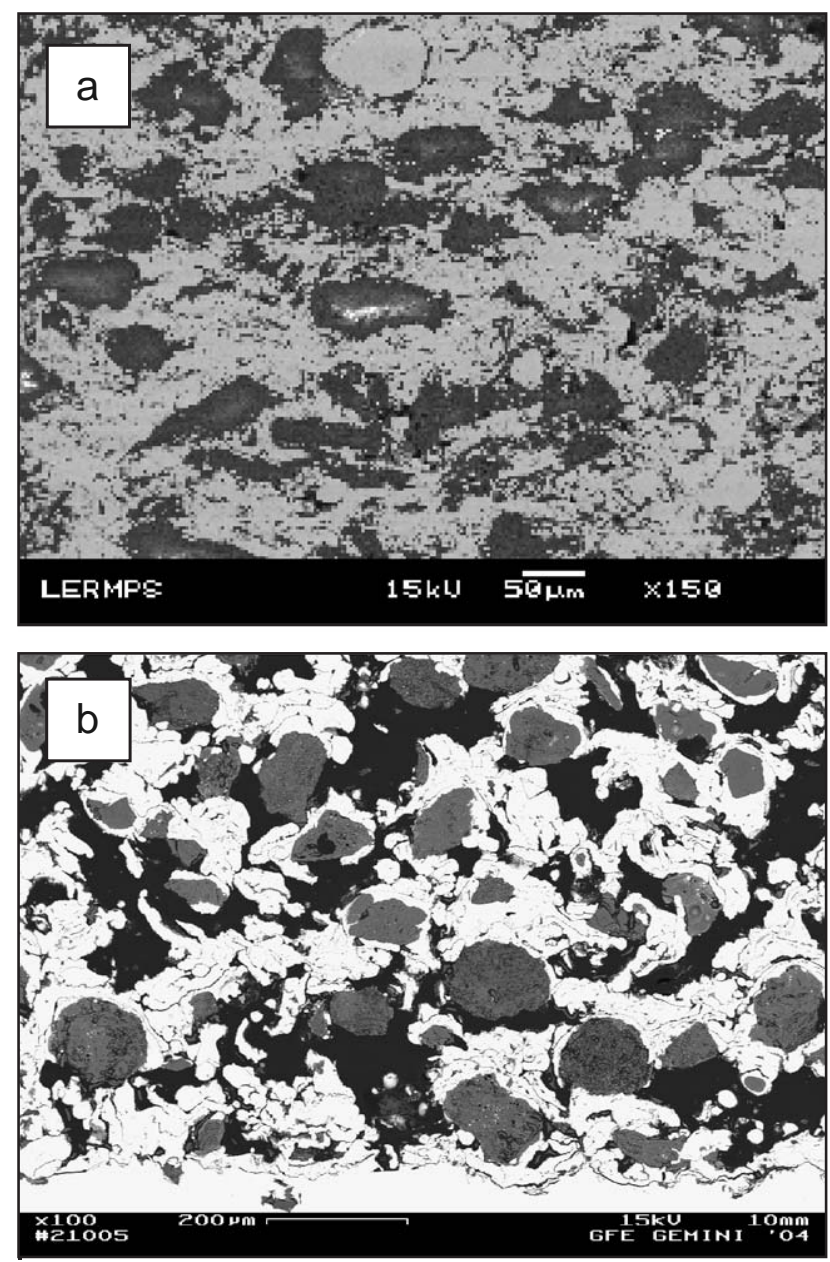

Fig. 1. Backscattered SEM micrograph of (a) the AlSi-hBN and (c) the NiCrAlBentonite coatings. 
Table 1

Volume fractions and input data for calculations of various structural elements of the coatings obtained by image analysis

AlSi-hBN coating

\begin{tabular}{lcclc}
\hline & & Metal matrix & Porosity & HBN particles \\
\hline Volume fraction & & $54 \pm 3 \%$ & $5 \pm 3 \%$ & $41 \pm 3 \%$ \\
Input data & $E$ & 17.0 & - & 7.2 \\
& $v$ & 0.33 & - & 0.1
\end{tabular}

NiCrAl-Bentonite coating

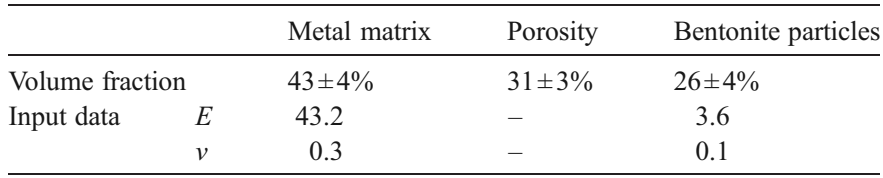

The Young modulus values are estimated using a correction coefficient of $24 \%$.

and porosity present in the coatings. For the AlSi-hBN coating (Fig. 1a), the metallic matrix (light grey contrast) and the boron nitride (deep grey contrast) can be clearly distinguished as the two major "structural elements". Apart from thin elongated porosity present at the intersplat metallic boundaries - a feature inherent to the thermal spray processing technique - the metal matrix is fairly dense. A small amount of porosity, estimated to be about $5 \%$, was located essentially at the metallic and boron nitride interfaces. The structure of the NiCrAl-Bentonite coating (Fig. 1b) consists of large bentonite particles (grey contrast) trapped in an opened network of lamellar metallic splats (white contrast). One of the major characteristics of this coating is the presence of a large amount of porosity. Its amount was estimated at about $31 \%$.

\section{Image analysis}

The selection of the appropriate magnifications for the images was done by selecting micrograph lengths corresponding, approximately, to 10 times the mean size of the structural element. This led us to adopt a higher magnification $(\times 150$ versus $\times 100)$ for the AlSi-hBN coating than for the NiCrAl-Bentonite one. For the metallic matrix that binds the particles, only the volume fraction was quantified. The detailed analysis of the porosity and second phase particle shape, size, distribution and orientation was done by constructing equivalent images of the micrographs. This construction was done by replacing each element on the micrograph by a corresponding ellipse that fits geometrically the particle morphology and orientation by means of three geometrical parameters: (i) shape factor: $F_{\mathrm{sh}}=A / B$. It corresponds to the ratio of the longest over the smallest axes. $F_{\mathrm{sh}}$ is representative of the particle elongation and is equal to unity for a circle; (ii) slope factor: $F_{\mathrm{sl}}=\alpha$. It corresponds to the orientation angle of the longest axes with respect to the horizontal direction. $F_{\mathrm{sl}}$ is representative of the orientation of the ellipses; and (iii) size factor: $F_{\mathrm{sz}}=A^{*} B$. it corresponds to the area of the ellipse with a factor of $4 / 3 \pi . F_{\text {sz }}$ contains the information about the average equivalent size of a particle.

The image analysis procedure was developed using a public domain software "imageJ" [17]. Following the choice of magnification, each image corresponds to a frame of $712 \times 484$ pixels for the AlSi-hBN coating and of $1280 \times 1024$ for the NiCrAl-Bentonite one. This gives a correspondence of $\sim 142$ pixels for $100 \mu \mathrm{m}$ in the case of the AlSi-hBN coating and of $\sim 112$ pixels for $100 \mu \mathrm{m}$ for the NiCrAl-Bentonite one. In each case, the image analysis procedure and the equivalent image generation were carried out on 15 micrographs. This led to a minimum of 1114 analysed objects and the normal object size $\left(3500 \mu^{2}\right)$ was therefore depicted using about 7057 pixels. Fig. 2 shows a typical equivalent image generated for NiCrAl-Bentonite coating. The procedure of equivalent image construction is detailed in [15] where it was shown how these "complexity-reduced" images provide a pertinent description of the structure and morphology of this kind of coatings.

\section{Calculation approach}

\subsection{Finite element model}

The obtained 2-D geometries are suitable for a finite element analysis. The mesh generation was done using the object-oriented code OOF [20]. Its meshing process produces decreasing element sizes close to the interfaces between two differently coloured regions, thereby permitting a good contour separation of the different phases present on the equivalent image. The finite element code is then used to build a bi-dimensional model for mechanical deformation of the coating. Artificial external strain is applied to the structure; the total energy of the system is then minimised with respect to the residual node displacements, thus leading to the overall system stress. Numerical tensile and shear testings are conducted by applying a series of strains with different amplitudes and solving the system in each case. Accordingly, one can plot stress versus strain curves for the system. This results in the parameters of a two-dimensional orthotropic and homogeneous material with a linear elastic law of behaviour. If we designate respectively $X$ and $Y$ the horizontal and vertical directions of the coating, we can

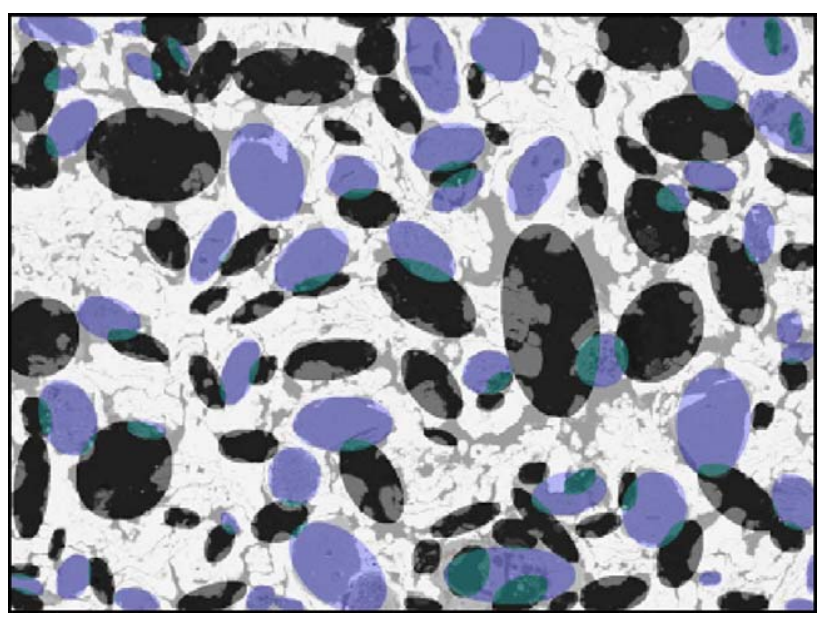

Fig. 2. Equivalent image generated for (a) NiCrAl-Bentonite and (b) AlSi-hBN coatings. 
define five parameters: Young modulus $E_{x}$ and $E_{y}$, Poisson coefficient $v_{x}$ and $v_{y}$ and a shear moduli $G$. The Hook law gives the relationship between the stresses $\sigma$ and the strains $\varepsilon$. In two-dimensional approximation we have:

$$
\left(\begin{array}{c}
\varepsilon_{x} \\
\varepsilon_{y} \\
\varepsilon_{x y}
\end{array}\right) \cdot\left(\begin{array}{ccc}
\frac{1}{E_{x}} & -\frac{v_{x}}{E_{x}} & 0 \\
-\frac{v_{y}}{E_{y}} & \frac{1}{E_{y}} & 0 \\
0 & 0 & \frac{1}{G}
\end{array}\right) \times\left(\begin{array}{c}
\sigma_{x} \\
\sigma_{y} \\
\sigma_{x y}
\end{array}\right)
$$

\subsection{Calculations results}

One key aspect at this point is the input data to be used in calculations. This includes the elastic properties of the constituent materials. The difficulty is that the consistency of matter within the coating is roughly modified compared to the massive materials because of the thermal spray process. In fact, the structure of the coating is lamellar and its density varies with the spraying conditions (in particular the temperature and the speed of cooling after melting). Consequently, the mechanical properties should be strongly reduced so that one can no longer deal with the measured values for massive materials. Accordingly, we set up a procedure of correction for the input values. We used a reference sample sprayed on a hastelloy substrate, whose Young modulus was measured by means of a bending test interpreted by an analytical calculation using the theory of the two-layer beams as described in [21]. The spraying conditions were set such that the coating is sufficiently dense (few porosities and no second phase particles). The calculations were then calibrated so that the input Young modulus provides the measured value. Fig. 3 shows the variation of the calculated value with respect to the input one. The measured value for the coating was of $38 \mathrm{GPa}$, that is to say $21 \%$ of the massif material Young modulus. At the same time, a ratio of $86 \%$ is calculated between input and calculated values. Thus, we come out with a ratio of about $24 \%$ to be used between the input value of the

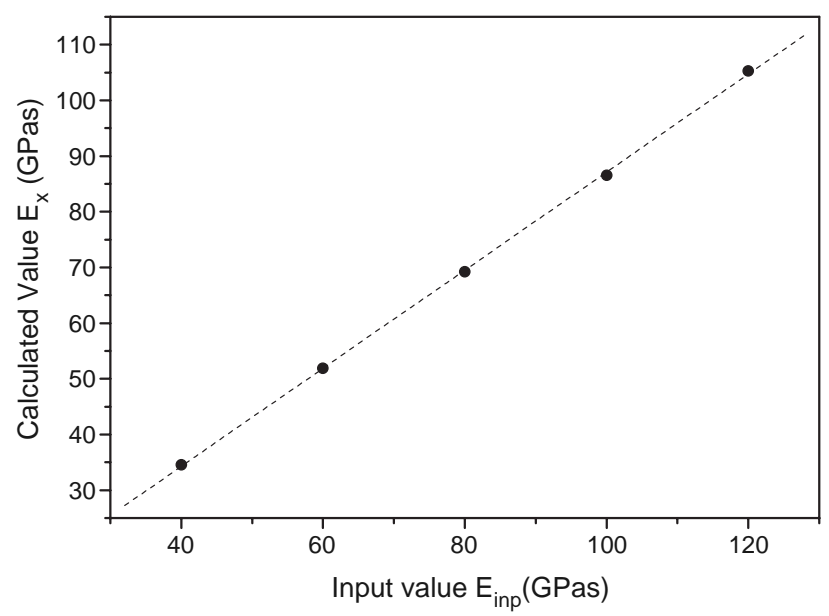

Fig. 3. Calculated versus input values of Young modulus for the reference coating.
Table 2

Calculated mechanical properties for the two coatings

\begin{tabular}{lcclll}
\hline & $E_{x}(\mathrm{GPa})$ & $E_{y}(\mathrm{GPa})$ & $G(\mathrm{GPa})$ & $v_{x}$ & $v_{y}$ \\
\hline $\mathrm{Sm} 314$ & 8.9 & 7.8 & 2.3 & 0.30 & 0.26 \\
$\mathrm{Sm} 320$ & 11.9 & 11.4 & 4.7 & 0.23 & 0.22 \\
\hline
\end{tabular}

Young modulus and that of the massive material in order for the calculations to provide the measured value (i.e. in order for the calculated value to match the measured one). At this level, we made the approximation that the same ratio can be used for the two studied coatings. Explicitly: a massive material looses its hardness to an amount of $76 \%$ when it is sprayed. Accordingly, we set up our input values presented in Table 1 .

Calculations were then carried on a set of equivalent images constructed on the basis of 15 micrographs. Table 2 resumes the calculated mechanical properties for both coatings. The magnitude of these values seems to be reasonable, but we did not find any measurements published about the modulus of this type of coatings. So our results remain at this time predictive. Accordingly, in parallel, a novel experimental testing method is being developed in order to assess the theoretical results. In this method, a polymer composite is used to produce water-soluble mould containing a cavity of required shape; abradable powder is sprayed into the mould cavity and the mould is dissolved leaving a freestanding coating. The abradable piece produced can be tested using standard mechanical or thermal analysis equipment. The rig test, currently underway, is aimed at producing data for coatings that will support the validation of our calculations.

\section{Conclusion}

This work intends to initiate a theoretical approach for the study of the microstructure of new abradable materials obtained by thermal spraying. The main objective was to develop a prediction strategy for structure improvement. The coatings under consideration are (i) an AlSi-hBN coating within which the hBN particles act as "lubricant" and (ii) a NiCrAl-Bentonite coating for which the bentonite is a dislocator.

A morphological description was used to reduce the complexity of the microstructure of coatings and an image analysis procedure has been established to quantify different geometrical parameters related to the shape, size and orientation distribution, of these structural elements. This description is pertinent for the analysis of this kind of coatings, and allows performing predictive calculation of the mechanical properties.

Finite element analysis was then presented which is intended to initiate a comprehensive study for the correlation between the microstructure of the coating and its mechanical law of behaviour. The model is used to perform calculations in the case of a classical two-dimensional orthotropic and homogeneous material with a linear elastic law of behaviour described by two Young's modulus, two Poison's ratios and one shear modulus. The results for both coatings appear to be reasonable, but no previous published work can be mentioned for 
comparison. Experimental measurements needed to validate our calculations are on track.

\section{Acknowledgments}

This study was funded by the European Commission under the FP5 Growth Program. It was completed within Seal-Coat on-going project, which involves the following partners: ESIL, Dublin/IE; Rolls-Royce plc, Derby/GB; MTU Aero Engines $\mathrm{GmbH}$, München/DE; Euromat GmbH, Heinsberg/DE; RWTH - Aachen University, Aachen/DE; Neomet Limited, Manchester/GB; Institute of Plasma Physics, Praha/ CZ; RALSA, Langreo/ES; UTBM - LERMPS, Belfort/FR.

\section{References}

[1] K.J. Melcher, J. Kypuros, Proceedings of the XVI International Symposium on Air Breathing Engines, Cleveland, OH, Aug. 31-Sep. 5, 2003, ISABE 2003-1102 (NASA/TM-2003-212627), 2003.

[2] A.M. Wallis, J.D. Denton, A.A.J. Demargne. Journal of Turbomachinery, $123,334$.

[3] B.M. Steinetz, R.C. Hendricks, J. Munson. NASA-TM 1998-206961.

[4] E.R. Novinski. ITSC 1991, Pittsburg, PA, USA. Ed. T.F. Bernecki, Pub. ASM International, Materials Parck, OH, USA. 451.

[5] R.K. Schmid, F. Ghasripoor, M. Dorfman, X. Wei, ITSC 2000, Montreal, Canada. Ed. C.C. Berndt, Pub. ASM International, Materials Park, OH, USA, 1087.

[6] M.O. Borel, A.R. Nicoll, H.W. Schäpfer, R.K. Schmid, Surf. Coat. Technol. 39/40 (1989) 117.
[7] J.M. Guilemany, J. Navarro, C. Lorenzana, S. Vizcaino, J.M. Miguel ITSC 2001, Singapore. C.C. Berndt, K.A. Khor and E.F. Lugscheider, Pub. ASM International, Materials Park, OH-USA. 1115.

[8] F. Ghasripoor, R.K. Schmid, M. Dorfman, L. Russo. ITSC 1998, Nice, France. C. Coddet, Pub. ASM International, Materials Park, OH-USA. 139.

[9] Y. Nava, Z. Mutasim, M. Coe. ITSC 2001, Singapore. Ed. C.C. Berndt, K.A. Khor, E.F. Lugscheider, Pub. A.S.M. International, Materials Park, O.H.-U.S.A. 119.

[10] Y.I. Maozhong, H. Baiyun, H. Jiawen, Wear 252 (2002) 9.

[11] M.Z. Yi, J.W. He, B.Y. Huang, H. Zhou, Wear 231 (1999) 47.

[12] S.C. Lim, M.F. Ashby, Acta Metall. 35 (1987) 1.

[13] M.F. Ashby, S.C. Lim, Scr. Metall. Mater. 24 (1990) 805.

[14] European Commission FP5 Growth Program. Abradable Seal Coatings and Claddings for Compressor Applications. Seal-Coat project No. GRD12001-40124.

[15] H.I. Faraoun, J.L. Seichepine, T. Grosdidier, H. Aourag, C. Coddet, J. Zwick, N. Hopkins. ITSC 2005, Basel, Switzerland. Ed. E. Lugscheider.

[16] P. Morrel, D.F. Bettridge, M.D. Greaves, M.R. Dorfman, L.D. Russo, C. Britton, K. Harrison. ITSC 1998, Nice, France. Ed. C. Coddet, Pub. ASM International, Materials Park, OH-USA. 1187.

[17] Wayne Rasband. National Institute of Health, USA.http://rsb.info.nih. gov/ij/.

[18] T.N. Rhys-Jones, Surf. Coat. Technol. 43/44 (1990) 402.

[19] T.N. Rhys-Jones, Surf. Coat. Technol. 42 (1990) 1.

[20] National Institute of Standards and Technology (NIST), OOF: Analysis of Real Material Microstructures (http://www.ctcms.nist.gov/oof/).

[21] H. Liao, Z. Feng, G. Jandin, C. Coddet, La Revue de Metallurgie-CIT/ Science et Genie des Materiaux, 2004, p. 109. 